\title{
Scoring rules and social choice properties: some characterizations
}

\author{
Bonifacio Llamazares · Teresa Peña
}

Received: date / Accepted: date

\begin{abstract}
In many voting systems, voters' preferences on a set of candidates are represented by linear orderings. In this context, scoring rules are well-known procedures to aggregate the preferences of the voters. Under these rules each candidate obtains a fixed number of points, $s_{k}$, each time he/she is ranked $k$ th by one voter and the candidates are ordered according to the total number of points they receive. In order to identify the best scoring rule to use in each situation, we need to know which properties are met by each of these procedures. Although some properties have been analyzed extensively, there are other properties that have not been studied for all scoring rules. In this paper we consider two desirable social choice properties, the Pareto-optimality and the immunity to the absolute loser paradox, and establish characterizations of the scoring rules that satisfy each of these specific axioms. Moreover, we also provide a proof of a result given by Saari and Barney (The Mathematical Intelligencer 25:17-31, 2003), where the scoring rules meeting reversal symmetry are characterized. From the results of characterization, we establish some relationships among these properties. Finally, we give a characterization of the scoring rules satisfying the three properties.
\end{abstract}

Keywords Scoring rules $\cdot$ Pareto-optimality $\cdot$ Absolute loser paradox $\cdot$ Reversal symmetry

JEL Classification D70 - D71

Bonifacio Llamazares, Teresa Peña

Departamento de Economía Aplicada, Instituto de Matemáticas (IMUVA), Universidad de Valladolid,

Avda. Valle de Esgueva 6, 47011 Valladolid, Spain.

Tel.: +34983186544

Fax: +34983423299

E-mail: boni@eco.uva.es, maitepe@eco.uva.es 


\section{Introduction}

Scoring rules ${ }^{1}$ are a class of voting systems where voters rank order the candidates from best to worst and each candidate obtains a fixed number of points, $s_{k}$, each time he/she is ranked $k$ th by one voter. The candidates who accumulate the most points are the winners.

The best known and studied scoring rules are the Borda rule and the $k$-approval voting rules. Nevertheless, there are numerous decisional contexts where other scoring rules are used. Among these contexts we can mention festivals like the Eurovision Song Contest, the FIFA Ballon d'Or Award or the choice of the Most Valuable Player of the National Basketball Association (MVP of the NBA). Also different scoring rules are used in sport competitions like the Formula One World Championship or the Motorcycle World Championship (in these events a voter is replaced by a race).

Many of the papers published on the topic of scoring rules are devoted to the study of social choice properties of these rules. Some specific properties have been studied extensively and we know exactly which scoring rules satisfy those axioms. For instance, it is well known that all scoring rules are anonymous, neutral, consistent and continuous (Young 1975), and satisfy the monotonicity property (Moulin 1988a), and participation ${ }^{2}$ (whenever ties are broken according to a fixed ordering of the candidates, see Moulin 1988b). Also, it has been proven that no scoring rule is Condorcet consistent (Fishburn 1974; Saari 2000b), that the plurality rule is the only scoring rule that verifies the absolute winner axiom ${ }^{3}$ (Lepelley 1992) and that the Borda rule is the only scoring rule that satisfies the Condorcet loser property (Smith 1973; Fishburn and Gehrlein 1976). A survey of these results can be found in Merlin (2003).

However there are other properties that have not been studied for all scoring rules and that are desirable from a social choice point of view. Among these properties we can cite the Pareto-optimality or the immunity to the absolute loser paradox (see, for instance, Felsenthal 2012; Plassmann and Tideman 2014). If we want to compare all scoring rules that are used in practice, in order to be able to make an assessment based on their respective strengths and weaknesses, we need to know which rules verify each of these axioms. For this purpose, in this paper we provide characterizations of the scoring rules that satisfy these specific social choice properties.

In order to obtain the candidates' score, scoring rules only need the number of votes obtained by each candidate in each $k$ th place. Instead of using these values, in this paper we consider, by each candidate, the number of votes obtained by him/her until the $k$ th place (called his/her cumulative standings). The cumulative standings allow us to deal with scoring rules without requiring that the scores are decreasing and, in this way, to obtain some results in an easier way. It is worth noting that cumulative standings have been previously used by Fishburn (1974), Stein et al. (1994), Green et al. (1996), Moulin (1988a, cap. 9), and Llamazares and Peña (2009, 2013), among others.

\footnotetext{
1 It is worth noting that the term scoring rules is also used, but with a different meaning, in some fields of experimental economic and decision theory.

2 Failing to satisfy this property is known as the no show paradox.

3 This axiom is called by the author the strong Condorcet winner property.
} 
The paper is organized as follows. In Section 2 we introduce scoring rules and show how the score obtained by each candidate can be expressed by using the cumulative standings obtained by him/her. Section 3 contains the main results of the paper. In this section we characterize the scoring rules that satisfy the Pareto-optimality property or that are immune to the absolute loser paradox. We also prove a characterization of the scoring rules satisfying reversal symmetry that appears, without an explicit proof, in Saari and Barney (2003). To end the section we provide some relationships among the properties studied and a characterization of the scoring rules meeting the three properties. Finally, Section 4 is devoted to conclusions. All proofs are in the Appendix.

\section{Scoring rules}

Throughout the paper, we will use the following notation: Given two vectors $\mathbf{x}, \mathbf{y} \in$ $\mathbb{R}^{k}, \mathbf{x} \geq \mathbf{y}$ denotes $x_{i} \geq y_{i}$ for all $i \in\{1, \ldots, k\} ; \mathbf{x}>\mathbf{y}$ means that $\mathbf{x} \geq \mathbf{y}$ and $\mathbf{x} \neq \mathbf{y}$; $\mathbf{x} \gg \mathbf{y}$ means that $x_{i}>y_{i}$ for all $i \in\{1, \ldots, k\}$; given $b \in \mathbb{R}$, b stands for the vector $(b, \ldots, b)$. Moreover, given $a \in \mathbb{R},\lfloor a\rfloor$ will denote the integer part of $a$, i.e., the largest integer smaller than or equal to $a$.

Let $\mathscr{A}=\left\{A_{1}, \ldots, A_{m}\right\}$ be a set of candidates, with $m \geq 3^{4}$. A weak order on $\mathscr{A}$ is a complete and transitive binary relation on $\mathscr{A}$. A linear order on $\mathscr{A}$ is a complete, transitive and antisymmetric binary relation on $\mathscr{A}$. A linear order on $\mathscr{A}$ where $A_{1}$ is the most preferred candidate, $A_{2}$ is the second most preferred candidate and so on will be written in the usual way as $A_{1} A_{2} \cdots A_{m-1} A_{m}$. Let $\mathscr{W}$ and $\mathscr{P}$ be, respectively, the sets of weak and linear orders on $\mathscr{A}$. A profile $\mathbf{p}$ is a vector of linear orders on $\mathscr{A}$ where each component represents the preferences of a voter.

A scoring vector $\mathbf{s}=\left(s_{1}, \ldots, s_{m}\right)$ is a vector $\mathbf{s} \in \mathbb{R}^{m}$, such that $s_{1} \geq s_{2} \geq \cdots \geq$ $s_{m}$ and $s_{1}>s_{m}$. Given the scoring vector $\mathbf{s}$, the scoring rule associated with $\mathbf{s}$ is a mapping from $\bigcup_{n>2} \mathscr{P}^{n}$ to $\mathscr{W}$ that, for each profile $\mathbf{p}$, assigns $s_{k}$ points to the $k$ th most preferred candidate of each voter and the candidates are ordered according to the total number of points they receive. So, the score obtained by candidate $A_{i}$ is $\sum_{k=1}^{m} s_{k} v_{k}^{i}$, where $v_{k}^{i}$ is the number of $k$ th place ranks that candidate $A_{i}$ occupies.

Note that, given a profile $\mathbf{p}$, scoring rules only use the number of first, second, $\ldots, m$ th ranks obtained by each candidate. Instead of using the number of ranks $v_{k}^{i}, k=1, \ldots, m$, in this paper we consider for each candidate $A_{i}$ his/her cumulative standings

$$
V_{k}^{i}=\sum_{l=1}^{k} v_{l}^{i}, \quad k=1, \ldots, m,
$$

which have been previously used in this context by Fishburn (1974), Stein et al. (1994), Green et al. (1996), Moulin (1988a, cap. 9) and Llamazares and Peña (2009, 2013), among others. Notice that the number of cumulative standings in the last place is always the number of voters in the profile $\mathbf{p}$.

\footnotetext{
4 When $m=2$, all the scoring rules coincide with the plurality rule.
} 
Taking into account that $v_{1}^{i}=V_{1}^{i}$ and $v_{k}^{i}=V_{k}^{i}-V_{k-1}^{i}$ for all $k \in\{2, \ldots, m\}$, we have

$$
\begin{aligned}
\sum_{k=1}^{m} s_{k} v_{k}^{i} & =s_{1} V_{1}^{i}+\sum_{k=2}^{m} s_{k}\left(V_{k}^{i}-V_{k-1}^{i}\right)=\sum_{k=1}^{m} s_{k} V_{k}^{i}-\sum_{k=2}^{m} s_{k} V_{k-1}^{i}=\sum_{k=1}^{m} s_{k} V_{k}^{i}-\sum_{k=1}^{m-1} s_{k+1} V_{k}^{i} \\
& =\sum_{k=1}^{m-1}\left(s_{k}-s_{k+1}\right) V_{k}^{i}+s_{m} V_{m}^{i}
\end{aligned}
$$

that is, the score obtained by candidate $A_{i}$ can be represented by using cumulative standings through the expression $\sum_{k=1}^{m} S_{k} V_{k}^{i}$, where $S_{k}=s_{k}-s_{k+1}$ for all $k \in$ $\{1, \ldots, m-1\}$ and $S_{m}=s_{m}$. From these relations it is easy to get that $s_{k}=\sum_{l=k}^{m} S_{l}$ for all $k \in\{1, \ldots, m\}$. Moreover, the conditions $s_{1} \geq s_{2} \geq \cdots \geq s_{m}$ are equivalent to $S_{k} \geq 0$ for all $k \in\{1, \ldots, m-1\}$, and the condition $s_{1}>s_{m}$ is equivalent to $S_{k}>0$ for some $k \in\{1, \ldots, m-1\}^{5}$.

On the other hand, it is well known that two scoring rules associated with the vectors $\mathbf{s}$ and $\mathbf{s}^{\prime}$ are equivalent (that is, they provide the same weak orders) if and only if there exist $a, b \in \mathbb{R}, a>0$, such that $\mathbf{s}^{\prime}=a \cdot \mathbf{s}+\mathbf{b}$. For this reason, in the literature of scoring rules (and specially in the case of three candidates) is usual to consider the following set of scoring vectors (see, for instance, Saari 1999, 2000a,b):

$$
\mathscr{S}^{*}=\left\{\mathbf{s} \in \mathbb{R}^{m} \mid s_{1}=1, s_{m}=0, s_{k} \geq s_{k+1}(k=1, \ldots, m-1)\right\} .
$$

Given $\mathbf{S}=\left(1, s_{2}, \ldots, s_{m-1}, 0\right) \in \mathscr{S}^{*}$, the scoring vector $\mathbf{S}$ associated with it is $\left(1-s_{2}, s_{2}-s_{3}, \ldots, s_{m-1}, 0\right)$; that is, it is a scoring vector with $L_{1}$-norm equals to 1 and $S_{m}=0$. Since $\sum_{k=1}^{m} S_{k} V_{k}^{i}=\sum_{k=1}^{m-1} S_{k} V_{k}^{i}$, we can take the following set of vectors ${ }^{6}$ :

$$
\mathscr{S}=\left\{\mathbf{S} \in \mathbb{R}^{m-1} \mid \mathbf{S}>\mathbf{0}, \sum_{k=1}^{m-1} S_{k}=1\right\} .
$$

So, given $\mathbf{S} \in \mathscr{S}$ and a profile $\mathbf{p}$, if we define the set $\mathscr{V}$ as

$$
\mathscr{V}=\left\{\left(V_{1}, \ldots, V_{m-1}\right) \in \mathbb{N}^{m-1} \mid 0 \leq V_{1} \leq \cdots \leq V_{m-1}\right\},
$$

and we denote by $\mathbf{V}^{i}$ the vector $\left(V_{1}^{i}, \ldots, V_{m-1}^{i}\right)$, that is, the cumulative standings ${ }^{7}$ of candidate $A_{i}$, then the score obtained by $A_{i}$ is $F_{\mathbf{S}}\left(\mathbf{V}^{i}\right)$, where $F_{\mathbf{S}}: \mathscr{V} \longrightarrow \mathbb{R}$ is defined by

$$
F_{\mathbf{S}}\left(V_{1}, \ldots, V_{m-1}\right)=\sum_{k=1}^{m-1} S_{k} V_{k}
$$

\footnotetext{
5 We will say that $\mathbf{s}$ and $\mathbf{S}$ are scoring vectors associated each other.

6 Reciprocally, given $\mathbf{S} \in \mathscr{S}$, the scoring vector $\mathbf{s} \in \mathscr{S}^{*}$ associated with it is

$$
\mathbf{s}=\left(1, S_{2}+\cdots+S_{m-1}, S_{3}+\cdots+S_{m-1}, \ldots, S_{m-1}, 0\right) .
$$
}

\footnotetext{
7 Although the cumulative standings of each candidate depend on the profile $\mathbf{p}$, in order to avoid cumbersome notation we shall omit $\mathbf{p}$ in the notation of these values when there is no possible confusion. When it will be necessary, we will use the notation $\mathbf{V}^{i}$ for the profile $\mathbf{p}, \mathbf{V}^{\prime i}$ for the profile $\mathbf{p}^{\prime}$ and so on (the same remark can be applied to the notation $v_{k}^{i}$ ).
} 
As in the case of the scoring vectors $\mathbf{s}$, when we use vectors of $\mathscr{S}$ we say that the scoring rule is associated with $\mathbf{S}$. Next, we represent by means of $F_{\mathbf{S}}$ some of the best-known scoring rules:

1. Plurality: A candidate receives one point each time he/she is ranked first by some voter and zero points for the other ranks. So, the candidates with the largest number of first ranks win. In the set of scoring vectors $\mathscr{S}$, plurality rule is obtained when $S_{1}=1$ and $S_{k}=0$ for all $k \in\{2, \ldots, m-1\}$; that is,

$$
F_{\mathbf{S}}\left(V_{1}, \ldots, V_{m-1}\right)=V_{1} \text {. }
$$

2. Antiplurality: A candidate receives zero points each time he/she is ranked last by some voter and one point for the other ranks. So, the candidates with the minor number of votes in the last position win. In the set of scoring vectors $\mathscr{S}$, antiplurality rule is obtained when $S_{k}=0$ for all $k \in\{1, \ldots, m-2\}$ and $S_{m-1}=1$; that is,

$$
F_{\mathbf{S}}\left(V_{1}, \ldots, V_{m-1}\right)=V_{m-1} .
$$

3. Borda rule: A candidate receives zero points each time he/she is ranked last by some voter, one point for being ranked next to last, up to $m-1$ points for being ranked first. In the set of scoring vectors $\mathscr{S}$, Borda rule is obtained when $S_{k}=$ $1 /(m-1)$ for all $k \in\{1, \ldots, m-1\}$; that is,

$$
F_{\mathbf{S}}\left(V_{1}, \ldots, V_{m-1}\right)=\frac{V_{1}+\cdots+V_{m-1}}{m-1},
$$

i.e., the score obtained by each candidate is the arithmetic mean of his/her cumulative standings.

4. k-approval voting: A candidate receives one point each time he/she is ranked in the $k$ first positions by some voter and zero points for the other ranks. So, the candidates with the largest number of votes in the $k$ first positions win. Obviously, plurality and antiplurality are special cases of $k$-approval voting (with $k=1$ and $k=m-1$, respectively). In the set of scoring vectors $\mathscr{S}, k$-approval voting is obtained when $S_{k}=1$ and $S_{i}=0$ for all $i \neq k$; that is,

$$
F_{\mathbf{S}}\left(V_{1}, \ldots, V_{m-1}\right)=V_{k}
$$

To finish this section, it is worth pointing out that the cumulative standings allow us to obtain an easy characterization of the candidates who are winners with all the scoring rules (see Fishburn 1974, Stein et al. 1994, and also, on this subject, Saari 1992 and Baharad and Nitzan 2006): Given a profile $\mathbf{p}$, the candidate $A_{i}$ is a winner with all scoring rules if and only if $\mathbf{V}^{i} \geq \mathbf{V}^{j}$ for all $j \neq i$.

\section{Social choice properties}

In this section we characterize the scoring rules that satisfy Pareto-optimality and that are immune to the absolute loser paradox. Moreover, we also provide a proof of a result given by Saari and Barney (2003), which characterizes the scoring rules satisfying reversal symmetry. Finally, in the last subsection we establish some relationships among the properties studied. 
3.1 Pareto-optimality

Pareto-optimality has been considered a basic property by some authors (see, for instance, Moulin 1988a, p. 233, or Felsenthal 2012). For this reason, the fulfilment of this property has been studied in the usual scoring rules. For instance, it is known that the Borda rule and, in general, all scoring rules with strictly decreasing weights are Pareto-optimal and that the antiplurality does not meet this property (see Moulin 1988a, p. 234). In the sequel we provide a characterization of all scoring rules that satisfy this axiom.

Definition 1 Let $\mathbf{p}$ be a profile.

1. A candidate $A_{i}$ dominates $A_{j}$ if all the voters strictly prefer $A_{i}$ to $A_{j}$.

2. A candidate $A_{i}$ is Pareto-optimal if there does not exist another candidate that dominates $A_{i}$.

Definition 2 A scoring rule is Pareto-optimal when, for all profile $\mathbf{p}$, all the winning candidates are Pareto-optimal.

This definition of Pareto-optimality has been used by Moulin (1988a, p. 233) and Nurmi (1999, p. 88), among others. Nevertheless, it is worth noting that some authors such as Fishburn (1974) and Felsenthal (2012) analyze the susceptibility of some voting systems to the following paradox (that they call the dominated-winner paradox or Pareto paradox): A no Pareto-optimal candidate may be a winner while a candidate that dominates him/her may not be. But scoring rules are immune to this paradox: Given a profile $\mathbf{p}$, if candidate $A_{i}$ dominates candidate $A_{j}$, then $\mathbf{V}^{i} \geq \mathbf{V}^{j}$. Therefore, for any $\mathbf{S} \in \mathscr{S}, F_{\mathbf{S}}\left(\mathbf{V}^{i}\right) \geq F_{\mathbf{S}}\left(\mathbf{V}^{j}\right)$ and, consequently, if $A_{j}$ is a winner, then so does $A_{i}$.

In the sequel we characterize the scoring rules that verify the Pareto-optimality property.

Theorem 1 Let $\mathbf{S} \in \mathscr{S}$. The following conditions are equivalent:

1. The scoring rule associated with $\mathbf{S}$ is Pareto-optimal.

2. $\mathbf{S} \gg \mathbf{0}$, or $\min _{k \in\{1, \ldots, m-1\}} S_{k}=0$ and

$$
\sum_{k=1}^{p-1}(m+k) S_{k}+\sum_{k=p+1}^{m-1} k S_{k}>m
$$

where $p=\min \left\{k \in\{1, \ldots, m-1\} \mid S_{k}=0\right\}$.

The previous theorem shows that the scoring rules associated with scoring vectors with non-null weights are Pareto-optimal. Therefore, the Borda rule is Paretooptimal. In the following corollary we show that the only Pareto-optimal $k$-approval voting is plurality rule.

Corollary $1 k$-approval voting is Pareto-optimal if and only if $k=1$. 
Next we express the result given in Theorem 1 in the usual set of scoring vectors $\mathscr{S}^{*}$.

Corollary 2 Let $\mathbf{s} \in \mathscr{S}^{*}$. The following conditions are equivalent:

1. The scoring rule associated with $\mathbf{s}$ is Pareto-optimal.

2. $1>s_{2}>\cdots>s_{m-1}>0$, or $\min _{k \in\{1, \ldots, m-1\}} s_{k}-s_{k+1}=0$ and

$$
\sum_{k=1}^{m-1} s_{k}>m \cdot s_{p}
$$

where $p=\min \left\{k \in\{1, \ldots, m-1\} \mid s_{k}=s_{k+1}\right\}$.

As we can see from the previous corollary, if the non-null scores of $\mathbf{s}$ are a strictly decreasing sequence then the scoring rule associated with $\mathbf{s}$ is Pareto-optimal. Thus, for instance, the scoring rules used at present in the Eurovision Song Context, the FIFA Ballon d'Or Award, the MVP of the NBA, the Formula One World Championship and the Motorcycle World Championship are Pareto-optimal.

\subsection{Absolute loser paradox}

Felsenthal (2012) reviews the main paradoxes afflicting voting procedures and points out the absolute loser paradox as an especially intolerable paradox. The performance of the usual scoring rules with regard to this property is known: Since the Borda rule satisfies the Condorcet loser property (see, for instance, Smith 1973, and Fishburn and Gehrlein 1976), it is also immune to the absolute loser paradox. We also know that the plurality rule is vulnerable to this paradox and that the antiplurality is immune to it (see, for instance, Plassmann and Tideman 2014). In the sequel we provide a characterization of the scoring rules that are immune to this paradox.

Definition 3 Let $\mathbf{p}$ be a profile. A candidate $A_{i}$ is the absolute loser if he/she is ranked last by an absolute majority of voters.

Definition 4 A scoring rule is immune to the absolute loser paradox if, for all profile p, the absolute loser, whenever he/she exists, is not a winning candidate.

Theorem 2 Let $\mathbf{S} \in \mathscr{S}$. The following conditions are equivalent:

1. The scoring rule associated with $\mathbf{S}$ is immune to the absolute loser paradox.

2. $\sum_{k=1}^{m-1} k S_{k} \geq \frac{m}{2}$.

From this result we can see that the Borda rule is immune to the absolute loser paradox and that, in the case of $k$-approval voting, the immunity appears when $k$ is greater than or equal to half the number of candidates.

\section{Corollary 3}


1. The Borda rule is immune to the absolute loser paradox.

2. $k$-approval voting is immune to the absolute loser paradox if and only if $k \geq m / 2$.

The characterization given in Theorem 2 is stated in the usual set of scoring vectors $\mathscr{S}^{*}$ as follows.

Corollary 4 Let $\mathbf{s} \in \mathscr{S}^{*}$. The following conditions are equivalent:

1. The scoring rule associated with $\mathbf{s}$ is immune to the absolute loser paradox.

2. $\sum_{k=1}^{m-1} s_{k} \geq \frac{m}{2}$.

It is easy to check that the scoring rules used at present in the Eurovision Song Context, the FIFA Ballon d'Or Award, the MVP of the NBA, the Formula One World Championship and the Motorcycle World Championship are vulnerable to the absolute loser paradox. For instance, in the case of the Formula One World Championship, we have that $\sum_{k=1}^{m-1} s_{k}=4.04$. Therefore, as the number of drivers for the 2013 season is 22 , the scoring rule is vulnerable to this paradox.

\subsection{Reversal symmetry}

Reversal symmetry was introduced by Saari (1994, p. 157) as a natural extension of neutrality.

Definition 5 A scoring rule satisfies reversal symmetry if, for all profile $\mathbf{p}$, reversing the ranking of each voter produces a reversed outcome.

A characterization of the scoring rules satisfying this property can be found in Saari and Barney (2003). However, these authors do not provide an explicit proof of this result. In order to give a proof of the characterization established by these authors, we introduce the notion of dual and self-dual vectors. These last scoring vectors allow us to get the wanted characterization.

\section{Definition 6}

1. Given $\mathbf{S}=\left(S_{1}, \ldots, S_{m-1}\right) \in \mathscr{S}$, the dual vector of $\mathbf{S}$ is $\mathbf{S}^{d}=\left(S_{m-1}, \ldots, S_{1}\right)$.

2. A vector $\mathbf{S} \in \mathscr{S}$ is self-dual if $\mathbf{S}=\mathbf{S}^{d}$.

Theorem 3 Let $\mathbf{S} \in \mathscr{S}$. The following conditions are equivalent:

1. The scoring rule associated with $\mathbf{S}$ satisfies reversal symmetry.

2. $\mathbf{S}$ is self-dual.

From this result it is obvious that Borda rule satisfies reversal symmetry and that $k$-approval voting satisfies this property if and only if $m$ is even and $k=m / 2$. Next we formulate Theorem 3 in the usual set of scoring vectors $\mathscr{S}^{*}$ (see Saari and Barney 2003).

Corollary 5 Let $\mathbf{s} \in \mathscr{S}^{*}$. The following conditions are equivalent:

1. The scoring rule associated with $\mathbf{s}$ satisfies reversal symmetry.

2. $1=s_{k+1}+s_{m-k}$ for all $k \in\{1, \ldots,\lfloor(m-1) / 2\rfloor\}$. 
3.4 Relationships among the properties

Once characterized the scoring rules that satisfy Pareto-optimality, reversal symmetry or that they are immune to the absolute loser paradox, it is possible to establish some relationships among these properties. So, it is straightforward to check that there is no relationship between Pareto-optimality and the absolute loser paradox. For instance, plurality rule is Pareto-optimal but it is vulnerable to the absolute loser paradox. And antiplurality rule is not Pareto-optimal but it is immune to the absolute loser paradox. However, if the scoring rule satisfies reversal symmetry, then it is immune to the absolute loser paradox, and it is Pareto-optimal when all the components of the scoring vector are non-null.

Theorem 4 Let $\mathbf{S} \in \mathscr{S}$. If the scoring rule associated with $\mathbf{S}$ satisfies reversal symmetry, then it is Pareto-optimal if and only if $\mathbf{S} \gg \mathbf{0}$.

Theorem $\mathbf{5}$ Let $\mathbf{S} \in \mathscr{S}$. If the scoring rule associated with $\mathbf{S}$ satisfies reversal symmetry, then it is immune to the absolute loser paradox.

Finally, next we give a characterization of the scoring rules meeting the three properties analyzed in the paper.

Corollary 6 Let $\mathbf{S} \in \mathscr{S}$. The scoring rule associated with $\mathbf{S}$ satisfies reversal symmetry, Pareto-optimality and it is immune to the absolute loser paradox if and only if $\mathbf{S}$ is self-dual and $\mathbf{S} \gg \mathbf{0}$.

\section{Conclusion}

In this paper we have considered two desirable social choice properties, Paretooptimality and immunity to the absolute loser paradox, and we have provided characterizations of the scoring rules that satisfy each of these axioms. We have also proven a characterization of the scoring rules satisfying reversal symmetry, which was given, without an explicit proof, by Saari and Barney (2003). After showing some relationships among these properties, we establish a characterization of the scoring rules satisfying the three properties. It is worth noting that our results have been achieved by using the number of cumulative standings obtained by each candidate. In this way, we have avoided having to use decreasing scoring vectors.

Acknowledgements The authors are grateful to the coordinating editor and an anonymous referee for valuable suggestions and comments. This work is partially supported by the Spanish Ministry of Economy and Competitiveness (Projects ECO2011-24200 and ECO2012-32178) and the Junta de Castilla y León (Consejería de Educación, Project VA066U13).

\section{A Proofs}

\section{A.1 Pareto-optimality}

By using contraposition, Theorem 1 is rewritten in an equivalent form in Theorem 6. Before stating and proving Theorem 6, we give two useful lemmas. 
Lemma 1 Let $\mathbf{p}$ be a profile. If the candidate $A_{i}$ is not Pareto-optimal, then $V_{1}^{i}=0$ and there exists another candidate $A_{j}$ with $V_{k}^{i} \leq V_{k-1}^{j}$ for all $k \in\{2, \ldots, m\}$ and $0=$ $V_{q}^{i}<V_{q}^{j}$, where $q=\min \left\{k \in\{1, \ldots, m-1\} \mid V_{k}^{j} \neq 0\right\}$. Moreover, $V_{k}^{i} \leq V_{k}^{j}$ for all $k \in\{1, \ldots, m-1\}$.

Proof. Given a profile $\mathbf{p}$, if $A_{i}$ is not Pareto-optimal, there exist another candidate $A_{j}$ that is preferred to $A_{i}$ by all voters, so $V_{1}^{i}=0$. On the other hand, when a voter places candidate $A_{i}$ in the $k$ th position, that voter will have placed $A_{j}$ in a higher scale position. Therefore, $V_{k}^{i} \leq V_{k-1}^{j}$ for all $k \in\{2, \ldots, m\}$. Furthermore, if $q \in\{1, \ldots, m-$ $1\}$ is the highest scale position achieved by candidate $A_{j}$, then $0=V_{q}^{i}<V_{q}^{j}$. Finally, given that $V_{k-1}^{j} \leq V_{k}^{j}$ for all $k \in\{2, \ldots, m-1\}$ and $V_{1}^{i}=0$, we have $V_{k}^{i} \leq V_{k}^{j}$ for all $k \in\{1, \ldots, m-1\}$.

Lemma 2 Let $\mathbf{S} \in \mathscr{S}$. If $\mathbf{S} \gg \mathbf{0}$, then the scoring rule associated with $\mathbf{S}$ is Paretooptimal.

Proof. Given a profile $\mathbf{p}$, we are going to prove that if a candidate $A_{i}$ is not Paretooptimal, then he/she is not a winning candidate. By Lemma 1, there exist a candidate $A_{j}$ with $V_{k}^{i} \leq V_{k}^{j}$ for all $k \in\{1, \ldots, m-1\}$ and $V_{q}^{i}<V_{q}^{j}$ for some $q \in\{1, \ldots, m-1\}$. Since $\mathbf{S} \gg \mathbf{0}$, we have $F_{\mathbf{S}}\left(\mathbf{V}^{i}\right)<F_{\mathbf{S}}\left(\mathbf{V}^{j}\right)$. Therefore, $A_{i}$ is not a winning candidate.

Theorem 6 Let $\mathbf{S} \in \mathscr{S}$. The following conditions are equivalent:

1. The scoring rule associated with $\mathbf{S}$ is not Pareto-optimal.

2. $\min _{k \in\{1, \ldots, m-1\}} S_{k}=0$ and

$$
\sum_{k=1}^{p-1}(m+k) S_{k}+\sum_{k=p+1}^{m-1} k S_{k} \leq m,
$$

where $p=\min \left\{k \in\{1, \ldots, m-1\} \mid S_{k}=0\right\}$.

Proof.

$1 \Rightarrow 2$ : If the scoring rule associated with $\mathbf{S}$ is not Pareto-optimal, then, by Lemma 2, we have $\min _{k \in\{1, \ldots, m-1\}} S_{k}=0$. Let $p=\min \left\{k \in\{1, \ldots, m-1\} \mid S_{k}=0\right\}$.

Moreover, we can find a profile $\mathbf{p}$ of $n$ voters such that one winning candidate, $A_{i}$, is not Pareto-optimal. By Lemma 1 , there exists a candidate $A_{j}$ with $V_{k}^{i} \leq V_{k}^{j}$ for all $k \in\{1, \ldots, m-1\}$. Therefore, $F_{\mathbf{S}}\left(\mathbf{V}^{i}\right) \leq F_{\mathbf{S}}\left(\mathbf{V}^{j}\right)$. Since $A_{i}$ is a winning candidate, we have $F_{\mathbf{S}}\left(\mathbf{V}^{i}\right)=F_{\mathbf{S}}\left(\mathbf{V}^{j}\right)$; that is

$$
\sum_{k=1}^{m-1} S_{k} V_{k}^{i}=\sum_{k=1}^{m-1} S_{k} V_{k}^{j} .
$$

Given that $S_{k} \geq 0$ and $V_{k}^{i} \leq V_{k}^{j}$ for all $k \in\{1, \ldots, m-1\}$, the previous equality is satisfied if and only if $S_{k} V_{k}^{i}=S_{k} V_{k}^{j}$ for all $k \in\{1, \ldots, m-1\}$. As $S_{k}>0$ for all $k<p$, we get $V_{k}^{i}=V_{k}^{j}$ for all $k<p$. If $q=\min \left\{k \in\{1, \ldots, m-1\} \mid V_{k}^{j} \neq 0\right\}$, then $0=V_{q}^{i}<$ $V_{q}^{j}$. Therefore, $q \geq p$ and, consequently, $V_{k}^{i}=0$ for all $k \in\{1, \ldots, p\}$. 
On the other hand, since $A_{i}$ is a winning candidate, we have

$$
\sum_{k=1}^{m-1} S_{k} V_{k}^{l} \leq \sum_{k=1}^{m-1} S_{k} V_{k}^{i}
$$

for all $l \in\{1, \ldots, m\}$. When we add, member to member, all the inequalities we have

$$
\sum_{l=1}^{m} \sum_{k=1}^{m-1} S_{k} V_{k}^{l}=\sum_{k=1}^{m-1} S_{k} \sum_{l=1}^{m} V_{k}^{l}=\sum_{k=1}^{m-1} S_{k} n k=n \sum_{k=1}^{m-1} k S_{k} \leq m \sum_{k=1}^{m-1} S_{k} V_{k}^{i}
$$

Since $V_{k}^{i}=0$ for all $k \in\{1, \ldots, p\}$, and $V_{k}^{i} \leq n$ for all $k \in\{p+1, \ldots, m-1\}$, we have

$$
n \sum_{k=1}^{m-1} k S_{k} \leq m n \sum_{k=p+1}^{m-1} S_{k}
$$

that is,

$$
\sum_{k=1}^{p-1} k S_{k}+\sum_{k=p+1}^{m-1} k S_{k} \leq m \sum_{k=p+1}^{m-1} S_{k}=m\left(1-\sum_{k=1}^{p-1} S_{k}\right)
$$

So, we get

$$
\sum_{k=1}^{p-1}(m+k) S_{k}+\sum_{k=p+1}^{m-1} k S_{k} \leq m
$$

$2 \Rightarrow 1:$ Let $\mathbf{S} \in \mathscr{S}$ such that $\min _{k \in\{1, \ldots, m-1\}} S_{k}=0$ and $\sum_{k=1}^{p-1}(m+k) S_{k}+\sum_{k=p+1}^{m-1} k S_{k} \leq$ $m$, where $p=\min \left\{k \in\{1, \ldots, m-1\} \mid S_{k}=0\right\}$. Consider $m-2$ voters and let $\mathbf{p}$ be a profile obtained by considering the forward cyclic list of orders ${ }^{8}$ generated by $A_{3} A_{4} \cdots A_{m}$ and by placing candidates $A_{1}$ and $A_{2}$ in the $p$ th and $(p+1)$ th positions, respectively; that is,

1 voter: $A_{3} A_{4} \cdots A_{p+1} A_{1} A_{2} A_{p+2} \cdots A_{m-1} A_{m}$

1 voter: $A_{4} A_{5} \cdots A_{p+2} A_{1} A_{2} A_{p+3} \cdots A_{m} A_{3}$

1 voter: $A_{5} A_{6} \cdots A_{p+3} A_{1} A_{2} A_{p+4} \cdots A_{3} A_{4}$

1 voter: $A_{m} A_{3} \cdots A_{p} A_{1} A_{2} A_{p+1} \cdots A_{m-2} A_{m-1}$.

As we can see in Table 1 , candidates $A_{3}, \ldots, A_{m}$ have the same cumulative standings; so they get the same score. The cumulative standings of candidates $A_{1}$ and $A_{2}$ differ only in the $p$ th cumulative standing, but $S_{p}=0$. Therefore, $A_{1}$ and $A_{2}$ obtain the same score. We calculate the scores of $A_{2}$ and $A_{3}$ :

$$
\begin{aligned}
& F_{\mathbf{S}}\left(\mathbf{V}^{2}\right)=\sum_{k=p+1}^{m-1}(m-2) S_{k}=(m-2) \sum_{k=p+1}^{m-1} S_{k}=(m-2)\left(1-\sum_{k=1}^{p-1} S_{k}\right), \\
& F_{\mathbf{S}}\left(\mathbf{V}^{3}\right)=\sum_{k=1}^{p-1} k S_{k}+\sum_{k=p+1}^{m-1}(k-2) S_{k} .
\end{aligned}
$$

8 According to Fishburn (1974), the forward cyclic list of orders generated by $A_{1} A_{2} \cdots A_{m}$ is the $m$-tuple of orders $\left(A_{1} A_{2} \cdots A_{m-1} A_{m}, A_{2} A_{3} \cdots A_{m} A_{1}, A_{3} A_{4} \cdots A_{1} A_{2}, \ldots, A_{m} A_{1} \cdots A_{m-2} A_{m-1}\right)$. 
Table 1 Cumulative standings of profile $\mathbf{p}$ (Proof of Theorem 6)

\begin{tabular}{cccccccccc}
\hline Candidate & $V_{1}^{i}$ & $V_{2}^{i}$ & $\cdots$ & $V_{p-1}^{i}$ & $V_{p}^{i}$ & $V_{p+1}^{i}$ & $V_{p+2}^{i}$ & $\cdots$ & $V_{m-1}^{i}$ \\
\hline$A_{1}$ & 0 & 0 & $\cdots$ & 0 & $m-2$ & $m-2$ & $m-2$ & $\cdots$ & $m-2$ \\
$A_{2}$ & 0 & 0 & $\cdots$ & 0 & 0 & $m-2$ & $m-2$ & $\cdots$ & $m-2$ \\
$A_{3}$ & 1 & 2 & $\cdots$ & $p-1$ & $p-1$ & $p-1$ & $p$ & $\cdots$ & $m-3$ \\
$A_{4}$ & 1 & 2 & $\cdots$ & $p-1$ & $p-1$ & $p-1$ & $p$ & $\cdots$ & $m-3$ \\
$\cdots$ & $\cdots$ & $\cdots$ & $\cdots$ & $\cdots$ & $\cdots$ & $\cdots$ & $\cdots$ & $\cdots$ & $\cdots$ \\
$A_{m}$ & 1 & 2 & $\cdots$ & $p-1$ & $p-1$ & $p-1$ & $p$ & $\cdots$ & $m-3$ \\
\hline
\end{tabular}

We now compare both scores:

$$
\begin{aligned}
F_{\mathbf{S}}\left(\mathbf{V}^{2}\right) \geq F_{\mathbf{S}}\left(\mathbf{V}^{3}\right) & \Leftrightarrow m-2-(m-2) \sum_{k=1}^{p-1} S_{k} \geq \sum_{k=1}^{p-1} k S_{k}+\sum_{k=p+1}^{m-1}(k-2) S_{k} \\
& \Leftrightarrow m-2 \geq \sum_{k=1}^{p-1}(m+k) S_{k}+\sum_{k=p+1}^{m-1} k S_{k}-2\left(\sum_{k=1}^{p-1} S_{k}+\sum_{k=p+1}^{m-1} S_{k}\right) \\
& \Leftrightarrow m \geq \sum_{k=1}^{p-1}(m+k) S_{k}+\sum_{k=p+1}^{m-1} k S_{k},
\end{aligned}
$$

where the last inequality is satisfied by hypothesis. Therefore, $A_{2}$ is a winning candidate but he/she is not Pareto-optimal because $A_{1}$ is preferred to $A_{2}$ by all voters. So, the scoring rule associated with $\mathbf{S}$ is not Pareto-optimal.

Proof of Corollary 1. If $\mathbf{S} \in \mathscr{S}$ is the scoring vector corresponding to the $k$-approval voting, the expression

$$
\sum_{l=1}^{p-1}(m+l) S_{l}+\sum_{l=p+1}^{m-1} l S_{l},
$$

where $p=\min \left\{l \in\{1, \ldots, m-1\} \mid S_{l}=0\right\}$, is equal to $m+1$ when $k=1$ and equal to $k$ when $k \geq 2$. From Theorem 1 we get the result.

Proof of Corollary 2. Let $\mathbf{s} \in \mathscr{S}^{*}$ and consider the scoring vector $\mathbf{S} \in \mathscr{S}$ associated with it. Given that $\mathbf{S} \gg \mathbf{0}$ if and only if $1>s_{2}>\cdots>s_{m-1}>0, S_{p}=0$ if and only if 


$$
\begin{aligned}
s_{p}= & s_{p+1}, \text { and } \\
\sum_{k=1}^{p-1}(m+k) S_{k}+\sum_{k=p+1}^{m-1} k S_{k}>m & \\
& \Leftrightarrow \sum_{k=1}^{p-1}(m+k)\left(s_{k}-s_{k+1}\right)+\sum_{k=p+1}^{m-1} k\left(s_{k}-s_{k+1}\right)>m \\
& \Leftrightarrow \sum_{k=1}^{p-1}(m+k) s_{k}-\sum_{k=2}^{p}(m+k-1) s_{k}+\sum_{k=p+1}^{m-1} k s_{k}-\sum_{k=p+2}^{m}(k-1) s_{k}>m \\
& \Leftrightarrow(m+1) s_{1}+\sum_{k=2}^{p-1} s_{k}-(m+p-1) s_{p}+(p+1) s_{p+1}+\sum_{k=p+2}^{m-1} s_{k}-(m-1) s_{m}>m \\
& \Leftrightarrow(m+1)+\sum_{k=2}^{p-1} s_{k}-(m-2) s_{p}+\sum_{k=p+2}^{m-1} s_{k}>m \\
& \Leftrightarrow 1+\sum_{k=2}^{p-1} s_{k}+\sum_{k=p+2}^{m-1} s_{k}>(m-2) s_{p} \\
& \Leftrightarrow \sum_{k=1}^{m-1} s_{k}>m \cdot s_{p},
\end{aligned}
$$

the result is obvious from Theorem 1 .

\section{A.2 Absolute loser paradox}

By using contraposition, Theorem 2 is rewritten in an equivalent form as follows.

Theorem 7 Let $\mathbf{S} \in \mathscr{S}$. The following conditions are equivalent:

1. The scoring rule associated with $\mathbf{S}$ is vulnerable to the absolute loser paradox.

2. $\sum_{k=1}^{m-1} k S_{k}<\frac{m}{2}$.

Proof.

$1 \Rightarrow 2$ : If the scoring rule associated with $\mathbf{S}$ is vulnerable to the absolute loser paradox, we can find a profile $\mathbf{p}$ such that one winning candidate, $A_{i}$, is the absolute loser. Therefore, he/she is ranked last by an absolute majority of voters and, consequently,

$$
V_{k}^{i} \leq n-(\lfloor n / 2\rfloor+1)=\lfloor(n-1) / 2\rfloor,
$$

for all $k \in\{1, \ldots, m-1\}$. On the other hand, since $A_{i}$ is a winning candidate, we have

$$
\sum_{k=1}^{m-1} S_{k} V_{k}^{l} \leq \sum_{k=1}^{m-1} S_{k} V_{k}^{i}
$$


Table 2 Cumulative standings of profile $\mathbf{p}$ (Proof of Theorem 2)

\begin{tabular}{ccccccc}
\hline Candidate & $V_{1}^{i}$ & $V_{2}^{i}$ & $V_{3}^{i}$ & $\cdots$ & $V_{m-2}^{i}$ & $V_{m-1}^{i}$ \\
\hline$A_{1}$ & $(m-1) r-1$ & $(m-1) r-1$ & $(m-1) r-1$ & $\cdots$ & $(m-1) r-1$ & $(m-1) r-1$ \\
$A_{2}$ & $r$ & $3 r-1$ & $5 r-1$ & $\cdots$ & $(2 m-5) r-1$ & $(2 m-3) r-1$ \\
$A_{3}$ & $r$ & $3 r$ & $5 r-1$ & $\cdots$ & $(2 m-5) r-1$ & $(2 m-3) r-1$ \\
$\cdots$ & $\cdots$ & $\cdots$ & $\cdots$ & $\cdots$ & $\cdots$ & $\cdots$ \\
$A_{m-1}$ & $r$ & $3 r$ & $5 r$ & $\cdots$ & $(2 m-5) r$ & $(2 m-3) r-1$ \\
$A_{m}$ & $r$ & $3 r$ & $5 r$ & $\cdots$ & $(2 m-5) r$ & $(2 m-3) r$ \\
\hline
\end{tabular}

for all $l \in\{1, \ldots, m\}$. When we add, member to member, all the inequalities we have

$$
\sum_{l=1}^{m} \sum_{k=1}^{m-1} S_{k} V_{k}^{l}=\sum_{k=1}^{m-1} S_{k} \sum_{l=1}^{m} V_{k}^{l}=\sum_{k=1}^{m-1} S_{k} n k=n \sum_{k=1}^{m-1} k S_{k} \leq m \sum_{k=1}^{m-1} S_{k} V_{k}^{i} \leq m\lfloor(n-1) / 2\rfloor
$$

Therefore,

$$
\sum_{k=1}^{m-1} k S_{k} \leq \frac{m}{n}\lfloor(n-1) / 2\rfloor<\frac{m}{2}
$$

$2 \Rightarrow 1:$ Since $\sum_{k=1}^{m-1} k S_{k}<m / 2$, there exists $r \in \mathbb{N}$ such that

$$
\sum_{k=1}^{m-1} k S_{k}<\frac{m}{2}-\frac{1}{2 r}
$$

Consider twice the forward cyclic list of orders generated by $A_{2} A_{3} \cdots A_{m}$. In the first forward cyclic list we place candidate $A_{1}$ in the first position and in the second forward cyclic list we place $A_{1}$ in the last position. Now we consider a profile $\mathbf{p}$ of $2(m-1) r-1$ voters where each order is considered $r$ times but the first one, which is considered $r-1$ times:

$$
\begin{array}{ll}
r-1 \text { voters: } & A_{1} A_{2} A_{3} \cdots A_{m-1} A_{m} \\
r \text { voters: } \quad & A_{1} A_{3} A_{4} \cdots A_{m} A_{2} \\
r \text { voters: } & A_{1} A_{4} A_{5} \cdots A_{2} A_{3} \\
\cdots & \cdots \cdots \cdots \cdots \\
r \text { voters: } & A_{1} A_{m} A_{2} \cdots A_{m-2} A_{m-1} \\
r \text { voters: } & A_{2} A_{3} \cdots A_{m-1} A_{m} A_{1} \\
r \text { voters: } & A_{3} A_{4} \cdots A_{m} A_{2} A_{1} \\
r \text { voters: } & A_{4} A_{5} \cdots A_{2} A_{3} A_{1} \\
\cdots & \cdots \cdots \cdots \\
r \text { voters: } & A_{m} A_{2} \cdots A_{m-2} A_{m-1} A_{1}
\end{array}
$$

In Table 2 we show the cumulative standings of candidates according to the profile p. Given that $\mathbf{V}^{m} \geq \mathbf{V}^{i}$ for all $i \in\{2, \ldots, m-1\}$, we have $F_{\mathbf{S}}\left(\mathbf{V}^{m}\right) \geq F_{\mathbf{S}}\left(\mathbf{V}^{i}\right)$ for all 
$i \in\{2, \ldots, m-1\}$. We now compare the scores of $A_{1}$ and $A_{m}$ :

$$
\begin{aligned}
F_{\mathbf{S}}\left(\mathbf{V}^{1}\right)>F_{\mathbf{S}}\left(\mathbf{V}^{m}\right) & \Leftrightarrow \sum_{k=1}^{m-1}((m-1) r-1) S_{k}>\sum_{k=1}^{m-1}(2 k-1) r S_{k} \\
& \Leftrightarrow(m-1) r-1>r \sum_{k=1}^{m-1}(2 k-1) S_{k} \\
& \Leftrightarrow m-1-\frac{1}{r}>\sum_{k=1}^{m-1}(2 k-1) S_{k} \\
& \Leftrightarrow m-\frac{1}{r}>\sum_{k=1}^{m-1}(2 k-1) S_{k}+\sum_{k=1}^{m-1} S_{k} \\
& \Leftrightarrow m-\frac{1}{r}>2 \sum_{k=1}^{m-1} k S_{k} \Leftrightarrow \frac{m}{2}-\frac{1}{2 r}>\sum_{k=1}^{m-1} k S_{k}
\end{aligned}
$$

where the last inequality is satisfied by the choice of $r$. Since $A_{1}$ is at the same time the winner and the absolute loser, $F_{\mathbf{S}}$ is vulnerable to the absolute loser paradox.

Proof of Corollary 3.

1. If $\mathbf{S} \in \mathscr{S}$ is the scoring vector corresponding to the Borda rule, we have

$$
\sum_{k=1}^{m-1} k S_{k}=\frac{1}{m-1} \sum_{k=1}^{m-1} k=\frac{1}{m-1} \frac{m(m-1)}{2}=\frac{m}{2} .
$$

So, the result is obvious from Theorem 2.

2. If $\mathbf{S} \in \mathscr{S}$ is the scoring vector corresponding to the $k$-approval voting, we have

$$
\sum_{l=1}^{m-1} l S_{l}=k
$$

Therefore, the result is obvious from Theorem 2 .

Proof of Corollary 4. Given $\mathbf{s} \in \mathscr{S}^{*}$, consider the scoring vector $\mathbf{S} \in \mathscr{S}$ associated with it. Since

$$
\sum_{k=1}^{m-1} k S_{k}=\sum_{k=1}^{m-1} k\left(s_{k}-s_{k+1}\right)=\sum_{k=1}^{m-1} k s_{k}-\sum_{k=2}^{m}(k-1) s_{k}=\sum_{k=1}^{m-1} s_{k}-(m-1) s_{m}=\sum_{k=1}^{m-1} s_{k}
$$

the result is obvious from Theorem 2 .

\section{A.3 Reversal symmetry}

Before we give the proof of Theorem 3, we previously establish two characterizations and a technical lemma. 
Proposition 1 The scoring rule associated with $\mathbf{S}$ satisfies reversal symmetry if and only if

$$
\begin{aligned}
F_{\mathbf{S}}\left(V_{1}^{i}, \ldots, V_{m-1}^{i}\right) & >F_{\mathbf{S}}\left(V_{1}^{j}, \ldots, V_{m-1}^{j}\right) \\
& \Leftrightarrow F_{\mathbf{S}}\left(n-V_{m-1}^{i}, \ldots, n-V_{1}^{i}\right)<F_{\mathbf{S}}\left(n-V_{m-1}^{j}, \ldots, n-V_{1}^{j}\right)
\end{aligned}
$$

for all profile $\mathbf{p}$ and all pair of candidates $A_{i}$ and $A_{j}$.

Proof. Given a profile $\mathbf{p}$, let $\left(V_{1}^{i}, \ldots, V_{m-1}^{i}\right)$ and $\left(V_{1}^{j}, \ldots, V_{m-1}^{j}\right)$ be the cumulative standings of candidates $A_{i}$ and $A_{j}$, respectively. It is easy to check that, when the profile $\mathbf{p}$ is reversed, the cumulative standings of $A_{i}$ and $A_{j}$ for this new profile are $\left(n-V_{m-1}^{i}, \ldots, n-V_{1}^{i}\right)$ and $\left(n-V_{m-1}^{j}, \ldots, n-V_{1}^{j}\right)$, respectively. From this, the result is obvious.

Proposition 2 The scoring rule associated with $\mathbf{S}$ satisfies reversal symmetry if and only if

$$
F_{\mathbf{S}}\left(\mathbf{V}^{i}\right)>F_{\mathbf{S}}\left(\mathbf{V}^{j}\right) \Leftrightarrow F_{\mathbf{S}^{d}}\left(\mathbf{V}^{i}\right)>F_{\mathbf{S}^{d}}\left(\mathbf{V}^{j}\right)
$$

for all profile $\mathbf{p}$ and all pair of candidates $A_{i}$ and $A_{j}$.

Proof. The proof is obvious from Proposition 1 and the following equivalence:

$$
\begin{aligned}
F_{\mathbf{S}}\left(n-V_{m-1}^{i}, \ldots, n-V_{1}^{i}\right) & <F_{\mathbf{S}}\left(n-V_{m-1}^{j}, \ldots, n-V_{1}^{j}\right) \\
& \Leftrightarrow \sum_{k=1}^{m-1} S_{k}\left(n-V_{m-k}^{i}\right)<\sum_{k=1}^{m-1} S_{k}\left(n-V_{m-k}^{j}\right) \\
& \Leftrightarrow n-\sum_{k=1}^{m-1} S_{k} V_{m-k}^{i}<n-\sum_{k=1}^{m-1} S_{k} V_{m-k}^{j} \\
& \Leftrightarrow \sum_{k=1}^{m-1} S_{k} V_{m-k}^{i}>\sum_{k=1}^{m-1} S_{k} V_{m-k}^{j} \\
& \Leftrightarrow \sum_{k=1}^{m-1} S_{m-k} V_{k}^{i}>\sum_{k=1}^{m-1} S_{m-k} V_{k}^{j} \\
& \Leftrightarrow F_{\mathbf{S}^{d}}\left(\mathbf{V}^{i}\right)>F_{\mathbf{S}^{d}}\left(\mathbf{V}^{j}\right) .
\end{aligned}
$$

Lemma 3 Given $l \in\{1, \ldots,\lfloor(m-1) / 2\rfloor\}$, there exist a profile $\mathbf{p}$ and candidates $A_{i}$ and $A_{j}$ such that $V_{l}^{i}=V_{l}^{j}+1, V_{m-l}^{i}=V_{m-l}^{j}-1$ and $V_{k}^{i}=V_{k}^{j}$ for all $k \neq l, m-l$.

Proof. Consider $m$ voters and the forward cyclic list of orders generated by the order $A_{1} A_{2} \cdots A_{m}$; that is,

1st voter: $A_{1} A_{2} \cdots A_{m-1} A_{m}$

2nd voter: $A_{2} A_{3} \cdots A_{m} A_{1}$

$m$ th voter: $A_{m} A_{1} \cdots A_{m-2} A_{m-1}$.

Now, in the first voter's preferences, we first change the candidates of positions $l$ and $l+1$ and, after that, we change the candidates of positions $m-l$ and $m-l+1$. Let $\mathbf{p}$ be the resulting profile. We distinguish two cases: 
Table 3 Cumulative standings of profile $\mathbf{p}$ (Proof of Lemma 3, case $l+1 \neq m-l$ )

\begin{tabular}{|c|c|c|c|c|c|c|c|c|c|}
\hline Candidate & $V_{1}^{i}$ & $\cdots$ & $V_{l}^{i}$ & $V_{l+1}^{i}$ & $\cdots$ & $V_{m-l}^{i}$ & $V_{m-l+1}^{i}$ & $\cdots$ & $V_{m-1}^{i}$ \\
\hline$A_{1}$ & 1 & $\ldots$ & $l$ & $l+1$ & $\cdots$ & $m-l$ & $m-l+1$ & $\cdots$ & $m-1$ \\
\hline$\ldots$ & $\ldots$ & $\ldots$ & $\ldots$ & $\ldots$ & $\ldots$ & $\ldots$ & $\ldots$ & $\ldots$ & $\ldots$ \\
\hline$A_{l}$ & 1 & $\ldots$ & $l-1$ & $l+1$ & $\ldots$ & $m-l$ & $m-l+1$ & $\ldots$ & $m-1$ \\
\hline$A_{l+1}$ & 1 & $\cdots$ & $l+1$ & $l+1$ & $\cdots$ & $m-l$ & $m-l+1$ & $\cdots$ & $m-1$ \\
\hline$\ldots$ & $\ldots$ & $\ldots$ & $\ldots$ & $\ldots$ & $\ldots$ & $\ldots$ & $\ldots$ & $\ldots$ & $\ldots$ \\
\hline$A_{m-l}$ & 1 & $\ldots$ & $l$ & $l+1$ & $\cdots$ & $m-l-1$ & $m-l+1$ & $\ldots$ & $m-1$ \\
\hline$A_{m-l+1}$ & 1 & $\ldots$ & $l$ & $l+1$ & $\cdots$ & $m-l+1$ & $m-l+1$ & $\cdots$ & $m-1$ \\
\hline$\ldots$ & $\cdots$ & $\cdots$ & $\cdots$ & $\ldots$ & $\cdots$ & $\ldots$ & $\ldots$ & $\cdots$ & $\cdots$ \\
\hline$A_{m}$ & 1 & $\cdots$ & $l$ & $l+1$ & $\cdots$ & $m-l$ & $m-l+1$ & $\cdots$ & $m-1$ \\
\hline
\end{tabular}

Table 4 Cumulative standings of profile $\mathbf{p}$ (Proof of Lemma 3, case $l+1=m-l$ )

\begin{tabular}{cccccccc}
\hline Candidate & $V_{1}^{i}$ & $\cdots$ & $V_{l}^{i}$ & $V_{l+1}^{i}$ & $V_{l+2}^{i}$ & $\cdots$ & $V_{m-1}^{i}$ \\
\hline$A_{1}$ & 1 & $\ldots$ & $l$ & $l+1$ & $l+2$ & $\cdots$ & $m-1$ \\
$\ldots$ & $\cdots$ & $\ldots$ & $\ldots$ & $\cdots$ & $\cdots$ & $\cdots$ & $\cdots$ \\
$A_{l}$ & 1 & $\ldots$ & $l-1$ & $l$ & $l+2$ & $\cdots$ & $m-1$ \\
$A_{l+1}$ & 1 & $\ldots$ & $l+1$ & $l+1$ & $l+2$ & $\cdots$ & $m-1$ \\
$A_{l+2}$ & 1 & $\ldots$ & $l$ & $l+2$ & $l+2$ & $\cdots$ & $m-1$ \\
$\ldots$ & $\cdots$ & $\cdots$ & $\ldots$ & $\cdots$ & $\cdots$ & $\cdots$ & $\cdots$ \\
$A_{m}$ & 1 & $\cdots$ & $l$ & $l+1$ & $l+2$ & $\cdots$ & $m-1$ \\
\hline
\end{tabular}

1. If $m-l \neq l+1$, then the first voter's order is

1st voter: $A_{1} \cdots A_{l-1} A_{l+1} A_{l} A_{l+2} \cdots A_{m-l-1} A_{m-l+1} A_{m-l} A_{m-l+2} \cdots A_{m}$, and the cumulative standings of profile $\mathbf{p}$ are given in Table 3 . As we can see, candidates $A_{l+1}$ and $A_{m-l+1}$ satisfy the thesis of the theorem.

2. If $m-l=l+1$, then the first voter's order is

1st voter: $A_{1} \cdots A_{l-1} A_{l+1} A_{l+2} A_{l} \cdots A_{m}$,

and the cumulative standings of profile $\mathbf{p}$ are given in Table 4.

As we can see, candidates $A_{l+1}$ and $A_{l+2}$ satisfy the thesis of the theorem.

\section{Proof of Theorem 3.}

$1 \Rightarrow 2$ : We are going to prove that if $\mathbf{S}$ is not self-dual then the scoring rule associated with $\mathbf{S}$ does not satisfy reversal symmetry. If $\mathbf{S}$ is not self-dual, then there exists $l \in\{1, \ldots,\lfloor(m-1) / 2\rfloor\}$ such that $S_{l} \neq S_{m-l}$. By Lemma 3, we can find a profile $\mathbf{p}$ and candidates $A_{i}$ and $A_{j}$ such that $V_{l}^{i}=V_{l}^{j}+1, V_{m-l}^{i}=V_{m-l}^{j}-1$ and $V_{k}^{i}=V_{k}^{j}$ for all $k \neq l, m-l$. For this profile and these candidates, we have

$$
\begin{aligned}
F_{\mathbf{S}}\left(\mathbf{V}^{i}\right)>F_{\mathbf{S}}\left(\mathbf{V}^{j}\right) & \Leftrightarrow \sum_{k=1}^{m-1} S_{k} V_{k}^{i}>\sum_{k=1}^{m-1} S_{k} V_{k}^{j} \\
& \Leftrightarrow S_{l} V_{l}^{i}+S_{m-l} V_{m-l}^{i}>S_{l} V_{l}^{j}+S_{m-l} V_{m-l}^{j} \\
& \Leftrightarrow S_{l}>S_{m-l},
\end{aligned}
$$


and

$$
\begin{aligned}
F_{\mathbf{S}^{d}}\left(\mathbf{V}^{i}\right)>F_{\mathbf{S}^{d}}\left(\mathbf{V}^{j}\right) & \Leftrightarrow \sum_{k=1}^{m-1} S_{m-k} V_{k}^{i}>\sum_{k=1}^{m-1} S_{m-k} V_{k}^{j} \\
& \Leftrightarrow S_{m-l} V_{l}^{i}+S_{l} V_{m-l}^{i}>S_{m-l} V_{l}^{j}+S_{l} V_{m-l}^{j} \\
& \Leftrightarrow S_{m-l}>S_{l} .
\end{aligned}
$$

Therefore, according to Proposition 2, the scoring rule associated with $\mathbf{S}$ does not satisfy reversal symmetry.

$2 \Rightarrow 1$ : Obvious by Proposition 2 .

Proof of Corollary 5. Given $\mathbf{s} \in \mathscr{S}^{*}$, consider the scoring vector $\mathbf{S} \in \mathscr{S}$ associated with it. Since

$$
\begin{aligned}
\mathbf{S}=\mathbf{S}^{d} & \Leftrightarrow S_{k}=S_{m-k} \text { for all } k \in\{1, \ldots,\lfloor(m-1) / 2\rfloor\} \\
& \Leftrightarrow s_{k}-s_{k+1}=s_{m-k}-s_{m-k+1} \text { for all } k \in\{1, \ldots,\lfloor(m-1) / 2\rfloor\} \\
& \Leftrightarrow s_{k}+s_{m-k+1}=s_{k+1}+s_{m-k} \text { for all } k \in\{1, \ldots,\lfloor(m-1) / 2\rfloor\} \\
& \Leftrightarrow 1=s_{k+1}+s_{m-k} \text { for all } k \in\{1, \ldots,\lfloor(m-1) / 2\rfloor\},
\end{aligned}
$$

the result is obvious from Theorem 3 .

A.4 Relationships among the properties

Proof of Theorem 4. By Theorem 1, it is sufficient to prove that if $\underset{k \in\{1, \ldots, m-1\}}{\min } S_{k}=0$, then

$$
\sum_{k=1}^{p-1}(m+k) S_{k}+\sum_{k=p+1}^{m-1} k S_{k} \leq m,
$$

where $p=\min \left\{k \in\{1, \ldots, m-1\} \mid S_{k}=0\right\}$. Since the scoring rule associated with $\mathbf{S}$ satisfies reversal symmetry, then, by Theorem $3, S_{k}=S_{m-k}$ for all $k \in\{1, \ldots, m-1\}$. Therefore, $S_{m-p}=S_{p}=0$ and $p \leq\lfloor m / 2\rfloor$. We distinguish two cases:

1. If $m-1$ is even, then

$$
\begin{aligned}
\sum_{k=1}^{p-1}(m+k) S_{k} & +\sum_{k=p+1}^{m-1} k S_{k} \\
& =\sum_{k=1}^{p-1}(m+k) S_{k}+\sum_{k=p+1}^{\frac{m-1}{2}} k S_{k}+\sum_{k=\frac{m+1}{2}}^{m-p-1} k S_{m-k}+\sum_{k=m-p+1}^{m-1} k S_{m-k} \\
& =\sum_{k=1}^{p-1}(m+k) S_{k}+\sum_{k=p+1}^{\frac{m-1}{2}} k S_{k}+\sum_{k=p+1}^{\frac{m-1}{2}}(m-k) S_{k}+\sum_{k=1}^{p-1}(m-k) S_{k} \\
& =2 m \sum_{k=1}^{p-1} S_{k}+m \sum_{k=p+1}^{\frac{m-1}{2}} S_{k} \leq 2 m \sum_{k=1}^{\frac{m-1}{2}} S_{k}=m \sum_{k=1}^{m-1} S_{k}=m .
\end{aligned}
$$


2. If $m-1$ is odd, we distinguish two cases:

(a) If $p=m / 2$, then

$$
\begin{aligned}
\sum_{k=1}^{\frac{m}{2}-1}(m+k) S_{k}+\sum_{k=\frac{m}{2}+1}^{m-1} k S_{k} & =\sum_{k=1}^{\frac{m}{2}-1}(m+k) S_{k}+\sum_{k=\frac{m}{2}+1}^{m-1} k S_{m-k} \\
& =\sum_{k=1}^{\frac{m}{2}-1}(m+k) S_{k}+\sum_{k=1}^{\frac{m}{2}-1}(m-k) S_{k} \\
& =2 m \sum_{k=1}^{\frac{m}{2}-1} S_{k}=m \sum_{k=1}^{m-1} S_{k}=m .
\end{aligned}
$$

(b) If $p<m / 2$, then

$$
\begin{aligned}
\sum_{k=1}^{p-1}( & m+k) S_{k}+\sum_{k=p+1}^{m-1} k S_{k} \\
= & \sum_{k=1}^{p-1}(m+k) S_{k}+\sum_{k=p+1}^{\frac{m}{2}-1} k S_{k}+\frac{m}{2} S_{\frac{m}{2}}+\sum_{k=\frac{m}{2}+1}^{m-p-1} k S_{m-k}+\sum_{k=m-p+1}^{m-1} k S_{m-k} \\
= & \sum_{k=1}^{p-1}(m+k) S_{k}+\sum_{k=p+1}^{\frac{m}{2}-1} k S_{k}+\frac{m}{2} S_{\frac{m}{2}}+\sum_{k=p+1}^{\frac{m}{2}-1}(m-k) S_{k}+\sum_{k=1}^{p-1}(m-k) S_{k} \\
= & 2 m \sum_{k=1}^{p-1} S_{k}+m \sum_{k=p+1}^{\frac{m}{2}-1} S_{k}+\frac{m}{2} S_{\frac{m}{2}} \leq m\left(2 \sum_{k=1}^{\frac{m}{2}-1} S_{k}+S_{\frac{m}{2}}\right)=m .
\end{aligned}
$$

Proof of Theorem 5. If the scoring rule associated with $\mathbf{S}$ satisfies reversal symmetry, then, by Theorem $3, S_{k}=S_{m-k}$ for all $k \in\{1, \ldots, m-1\}$. We distinguish two cases:

1. If $m-1$ is even, then

$$
\sum_{k=1}^{m-1} k S_{k}=\sum_{k=1}^{\frac{m-1}{2}} k S_{k}+\sum_{k=\frac{m+1}{2}}^{m-1} k S_{m-k}=\sum_{k=1}^{\frac{m-1}{2}} k S_{k}+\sum_{k=1}^{\frac{m-1}{2}}(m-k) S_{k}=m \sum_{k=1}^{\frac{m-1}{2}} S_{k}=\frac{m}{2} .
$$

2. If $m-1$ is odd, then

$$
\begin{aligned}
\sum_{k=1}^{m-1} k S_{k} & =\sum_{k=1}^{\frac{m}{2}-1} k S_{k}+\frac{m}{2} S_{\frac{m}{2}}+\sum_{k=\frac{m}{2}+1}^{m-1} k S_{m-k}=\sum_{k=1}^{\frac{m}{2}-1} k S_{k}+\frac{m}{2} S_{\frac{m}{2}}+\sum_{k=1}^{\frac{m}{2}-1}(m-k) S_{k} \\
& =m \sum_{k=1}^{\frac{m}{2}-1} S_{k}+\frac{m}{2} S_{\frac{m}{2}}=\frac{m}{2}\left(2 \sum_{k=1}^{\frac{m}{2}-1} S_{k}+S_{\frac{m}{2}}\right)=\frac{m}{2} \sum_{k=1}^{m-1} S_{k}=\frac{m}{2} .
\end{aligned}
$$

Therefore, in both cases, $\sum_{k=1}^{m-1} k S_{k}=m / 2$ and, by Theorem 2 , the scoring rule associated with $\mathbf{S}$ is immune to the absolute loser paradox.

Proof of Corollary 6. It is obvious from Theorems 1, 3, 4 and 5. 


\section{References}

Baharad, E., \& Nitzan, S. (2006). On the selection of the same winner by all scoring rules. Social Choice and Welfare, 26, 597-601.

Felsenthal, D. S. (2012). Review of paradoxes afflicting procedures for electing a single candidate. In D. S. Felsenthal \& M. Machover (Eds.), Electoral Systems: Paradoxes, Assumptions, and Procedures (pp. 19-91). Berlin: Springer.

Fishburn, P. C. (1974). Paradoxes of voting. American Political Science Review, 68, 537-546.

Fishburn, P. C., \& Gehrlein, W. V. (1976). Borda's rule, positional voting, and Condorcet's simple majority principle. Public Choice, 28, 79-88.

Green, R. H., Doyle, J. R., \& Cook, W. D. (1996). Preference voting and project ranking using DEA and cross-evaluation. European Journal of Operational Research, 90, 461-472.

Lepelley, D. (1992). Une caractérisation du vote à la majorité simple. RAIRO - Operations Research, 26, 361-365.

Llamazares, B., \& Peña, T. (2009). Preference aggregation and DEA: An analysis of the methods proposed to discriminate efficient candidates. European Journal of Operational Research, 197, 714-721.

Llamazares, B., \& Peña, T. (2013). Aggregating preferences rankings with variable weights. European Journal of Operational Research, 230, 348-355.

Merlin, V. (2003). The axiomatic characterizations of majority voting and scoring rules. Mathématiques et Sciences Humaines, 163, 87-109.

Moulin, H. (1988a). Axioms of Cooperative Decision Making. Cambridge: Cambridge University Press.

Moulin, H. (1988b). Condorcet's principle implies the no show paradox. Journal of Economic Theory, 45, 53-64.

Nurmi, H. (1999). Voting Paradoxes and How to Deal with Them. Berlin: Springer-Verlag.

Plassmann, F., \& Tideman, T. (2014). How frequently do different voting rules encounter voting paradoxes in three-candidate elections? Social Choice and Welfare, 42, 31-75.

Saari, D. G. (1992). Millions of election outcomes from a single profile. Social Choice and Welfare, 9 , 277-306.

Saari, D. G. (1994). Geometry of Voting. Berlin: Springer-Verlag.

Saari, D. G. (1999). Explaining all three-alternative voting outcomes. Journal of Economic Theory, 87, 313-355.

Saari, D. G. (2000a). Mathematical structure of voting paradoxes I: Pairwise votes. Economic Theory, 15 , $1-53$.

Saari, D. G. (2000b). Mathematical structure of voting paradoxes II: Positional voting. Economic Theory, $15,55-102$.

Saari, D. G., \& Barney, S. (2003). Consequences of reversing preferences. The Mathematical Intelligencer, $25,17-31$.

Smith, J. H. (1973). Aggregation of Preferences with Variable Electorate. Econometrica, 41, 1027-1041.

Stein, W. E., Mizzi, P. J., \& Pfaffenberger, R. C. (1994). A stochastic dominance analysis of ranked voting systems with scoring. European Journal of Operational Research, 74, 78-85.

Young, H. P. (1975). Social choice scoring functions. SIAM Journal on Applied Mathematics, 28, 824-838. 